\title{
Enneking Stage III
}

National Cancer Institute

\section{Source}

National Cancer Institute. Enneking Stage III. NCI Thesaurus. Code C146705.

A stage for malignant musculoskeletal tumors characterized by any grade, any site, and regional or distant metastasis (M1). 